\title{
Understanding High-Pressure Formation of Topologically Non-trivial Intermetallic Compounds via In-Situ X-ray Diffraction
}

\author{
A Tamerius ${ }^{1}$ \\ ${ }^{1}$ Northwestern University, Evanston, IL \\ alexandratamerius2021@u.northwestern.edu
}

The investigation of topologically non-trivial surface states is at the forefront of condensed matter physics and material science. Of particular interest is the concurrence of non-trivial topology with superconductivity which has sparked both fundamental questions about the intersection of these phenomena and revealed potential applications in quantum computing. Although the extensive exploration of temperature-composition phase spaces has enabled experimental realization of topologically non-trivial compounds, very few superconduct. This paucity of topologically non-trivial superconductors creates an impetus for alternate synthetic routes to discover new topologically non-trivial superconductors. A key criterion for accessing non-trivial topology is the combination of a large degree of spin-orbit coupling and mobile electrons in the correct symmetry. To realize new topologically non-trivial superconductor candidates, we chose to combine lead, the largest, non-radioactive, main-group element, as a source of spin-orbit coupling with transition metals as a source of mobile electrons. As lead reacts with latetransition metals to form superconducting intermetallic compounds and many transition-metal-lead binary compounds have suitable symmetry for non-trivial topology, we chose to target binary compounds that combine late-transition metals with lead as promising candidates for topologically non-trivial superconductivity. Among these binary systems, a few, such as, $\mathrm{Ni}-\mathrm{Pb}$, had no thermodynamically stable compounds reported despite thorough investigation of their binary temperature-composition phase space. By combining high-pressure, hightemperature synthesis with in-situ and ex-situ X-ray diffraction techniques, we synthesized and crystallographically characterized the first intermetallic compound in the $\mathrm{Ni}-\mathrm{Pb}$ system, $\mathrm{Ni}_{3} \mathrm{~Pb}_{2}$. The pseudo-hexagonal symmetry within the modulated $\mathrm{Ni}_{3} \mathrm{~Pb}_{2}$ structure and the Dirac cones predicted at the Fermi energy of the band structure suggest $\mathrm{Ni}_{3} \mathrm{~Pb}_{2}$ may exhibit non-trivial topology. Moreover, $\mathrm{Ni}_{3} \mathrm{~Pb}_{2}$ is recoverable to ambient conditions enabling further investigation of its electronic properties. Here, we present the high-pressure synthesis of $\mathrm{Ni}_{3} \mathrm{~Pb}_{2}$, our insight into its formation, and our progress investigating its properties.

Acta Cryst. (2020). A76, a141 